\title{
Bronchopulmonary Dysplasia and Ureaplasma: What Do We Know So Far?
}

\author{
Nicole de la Haye, M.D. ${ }^{*}$, Matthias C. Hütten, M.D., , , Steffen Kunzmann, M.D. ${ }^{\S}$, Boris W. Kramer, M.D., ${ }^{*}$, ॥ \\ Department of Pediatrics*, Maastricht University Medical Center (MUMC), Maastricht, The Netherlands \\ School of Oncology and Developmental Biology (GROW) ${ }^{\dagger}$, Maastricht, The Netherlands \\ Division of Neonatology, Department of Pediatrics ${ }^{\ddagger}$, Würzburg University Hospital, Würzburg, Germany \\ School for Mental Health and Neuroscience ${ }^{\S}$, Maastricht University, Maastricht, The Netherlands \\ Department of Neonatology and Pediatric Intensive Care Medicine", Bürgerhospital Frankfurt am Main, Germany
}

\section{ABSTRACT}

Bronchopulmonary dysplasia (BPD) is the most common morbidity of prematurity. BPD is a chronic respiratory disease related to lung-injury during the primary course of critical lung disease such as respiratory distress syndrome or when abnormal development of the preterm lung occurs. Abnormal lung development not only results from primary lung injury in the first days after birth, but also secondary injury through abnormal repair resulting in arrested and abnormal alveolarization, fibrosis and pulmonary vascular dysgenesis. Chorioamnionitis is a risk factor that plays an important role in the development of BPD. Ureaplasma subspecies (spp.) are the most common isolated organisms from chorioamniotic tissue after premature births. Therefore Ureaplasma spp. appear to play an important role in the development of BPD, and treatment or prophylactic treatment of these infections or colonization may reduce the incidence, morbidity and mortality of BPD. Ureaplasma spp. infections are challenging not only to treat, but also to diagnosis in a timely manner. This review summarizes the current state of treatment and new developments in the treatment of Ureaplasma exposure in premature infants.

Key Words: Bronchopulmonary dysplasia, Ureaplasma, Chorioamnionitis, Macrolides, Solithromycin, Azithromycin

\section{INTRODUCTION}

Bronchopulmonary dysplasia (BPD) is the most common morbidity of prematurity ${ }^{1)}$. $\mathrm{BPD}$ is a chronic respiratory disease related to lung-injury during the primary course of critical lung disease such as respiratory distress syndrome or abnormal lung development in utero and postnatally. Abnormal lung development occurs not only through primary lung injury in the first days after birth, but also secondary lung injury through abnormal repair resulting in arrested and abnormal alveolarization, fibrosis and pulmonary vascular
Received: 15 August 2016

Revised: 30 November 2016

Accepted: 7 December 2016

Correspondence to: Boris W. Kramer Department of Pediatrics, Maastricht University Medical Center, P. Debyelaan 25, 6202 AZ Maastricht, The Netherlands

Tel: +31-43-387-4202

Fax: +31-43-387-5246

E-mail: b.kramer@ maastrichtuniversity.nl

Copyright(c)

By Korean Society of Neonatology.

All right reserved.

This is an Open-Access article distributed under the terms of the Creative Commons Attribution Non-Commercial License (http://creativecommons.org/licenses/ by-nc/4.0), which permits unrestricted non-commercial use, distribution, and reproduction in any medium, provided the original work is properly cited. 
dysgenesis. The definition and classification of BPD have changed since it was first described in 1967 by Northway et al. ${ }^{2)}$. Currently, the most frequently used definition of BPD is as persistent oxygen dependency for up to 28 days of life. The severity of BPD is more accurately predicted by the oxygen dependence at 36 weeks' postmenstrual age in infants born younger than 32 weeks gestational age and at 56 days of age in infants with older gestational age.

BPD is a multifactorial disease. These multifactorial influences occur postnatally as mechanical ventilation, oxygen toxicity, and sepsis. In addition to these postnatal factors, prenatal exposures may prime the lung for BPD development, such as through chorioamnionitis and maternal glucocorticosteroid administration ${ }^{3}$. These different factors may affect the development and severity of BPD in individual premature infants ${ }^{1,4)}$.

Despite ongoing research on the development and treatment of BPD, treatment options are limited. Therefore BPD remains a major health issue in preterm infants, as BPD affects not only health care costs, but more importantly, the subsequent growth and neurodevelopment of the affected preterm baby ${ }^{5)}$. There is growing evidence that prenatal factors influence the development of BPD, and prophylactic therapy to prevent BPD may play a key role in the future. However, limited research data are available.

This review summarizes the current state of treatment and new developments in the treatment of Ureaplasma exposure in premature infants.

\section{UREAPLASMA AND BPD - EPIDEMIOLOGY}

Chorioamnionitis is one risk factor that plays an important role in the development of $\mathrm{BPD}^{6}$. Ureaplasma spp. are the most common isolated organisms from chorioamniotic tissue after premature births ${ }^{7}$. Intra-uterine colonization and infections with Ureaplasma spp. led to altered lung development, prolonged inflammatory response and increased fibrotic reaction in animal models ${ }^{7-10)}$. In a previous meta-analysis, Wang and Schelonka, suggested that there is an association between postnatal Ureaplasma infection and BPD development ${ }^{9,11)}$. Therefore, Ureaplasma spp. appear to play an important role in the development of BPD, and thus treatment or prophylactic treatment of these infections or colonization may reduce the incidence, morbidity and mortality of BPD.
Ureaplasma has been detected not only in the placentas and amniotic fluid, but also in the cord blood, cerebrospinal fluid, respiratory secretions, gastric aspirates, brain and lung tissue of preterm infants, and therefore is associated with ad verse pregnancy and neonatal morbidity of prematurity ${ }^{9,12)}$. Its clinical influence and relevance on the development of BPD and respiratory tract colonization has been demonstrated in four meta analyses in the past 20 years ${ }^{7,9,11,13)}$. Further studies reported that the incidence of colonization ranged from rates 20 to $45 \%$ in very low birth weight infants ${ }^{14)}$. A high incidence has been reported, and it has been shown that the risk of colonization increases with decreasing gestational age. Sixty-five percent of infants less than 26 weeks' gestation were culture- or PCR-positive one or more times during the first month of life compared to $31 \%$ of infants 26 or more weeks gestational age ${ }^{12,15}$.

\section{UREAPLASMA SPP - CHARACTERISTICS}

Among Ureaplasma spp., $U$. parvum and $U$. urealyticum are the most commonly isolated pathogens from infected placentas and amniotic fluid. $U$. parvum is isolated in $63 \%$ of respiratory cultures of neonatal respiratory samples at all ages. $U$. parvum serovars 3 and 6 account for $96 \%$ of these isolates. For $U$. urealyticum is serovar 11 the most common type isolated ${ }^{12)}$. There is no difference in morbidity between $U$. parvum (serovar 1, 3, 6, and 14 ) and $U$. urealyticum (serovar 2, 4, 5, 7-13) ${ }^{10,12)}$. There is no difference in the prevalence of either species between infants with or without $\mathrm{BPD}^{13,16)}$. Thus, Ureaplasma spp. act as pathogens, but there is no difference between species concerning the clinical outcome (Figure 1).

\section{UREAPLASMA AND PATHOPHYSIOLOGY}

How do these pathogens function in the pathogenesis of BPD? Ureaplasma spp. adhere to the human mucosal surfaces of the genitourinary tract in adults and respiratory tract in newborns. Ureaplasma spp. may contribute to cell damage of pneumocytes by virulent factors. First, Ureaplasma spp. activate different Tolllike-receptors as observed in the results of in vitro studies ${ }^{17,18)}$. Ureaplasma spp. induce the production of tumor necrosis factoralpha, interleukin (IL)-6 and apoptosis of human type 2 lung epithelial cells and lung macrophages. Second, Ureaplasma 


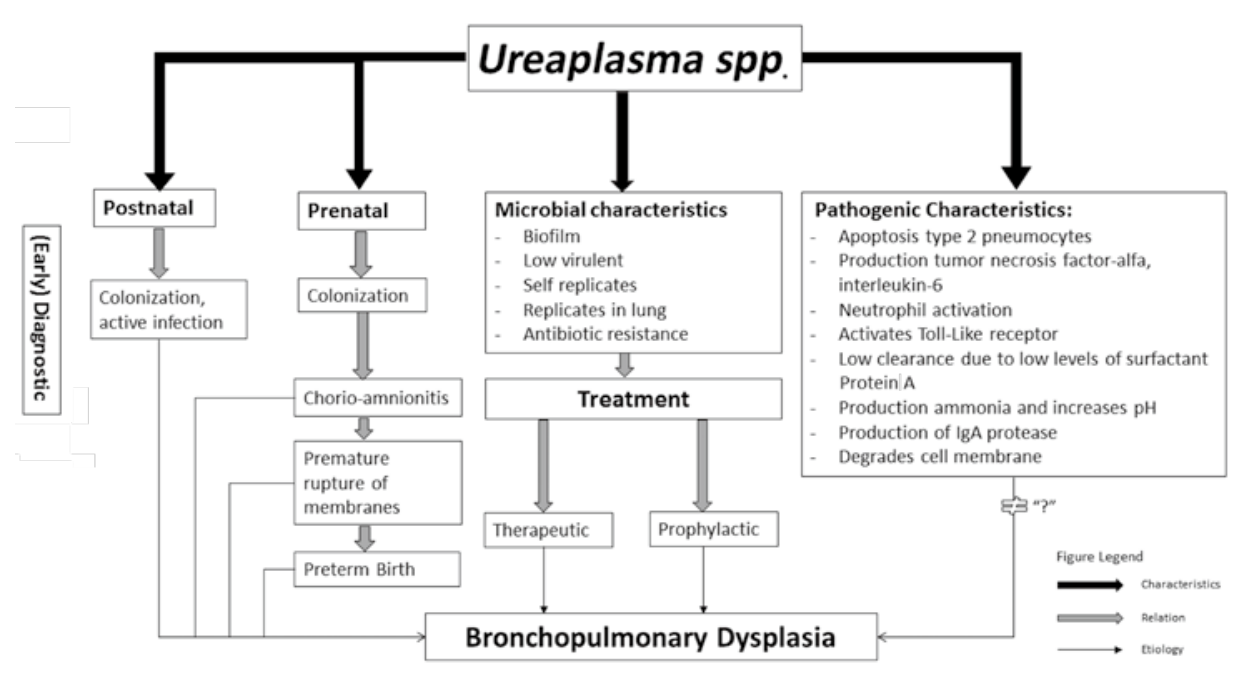

Figure 1. Schematic overview of characteristics and pathogenic behavior of Ureaplasma subspecies leading to bronchopulmonary dysplasia. Abbreviation: Ureaplasma spp., Ureaplasma subspecies.

spp. are known to induce antibody production in both humans and animals ${ }^{19)}$. In vitro models, it also has been shown that surfactant protein A (SP-A) enhances phagocytosis and killing of Ureaplasma, which may enhance delayed clearing of Ureaplasma from the lungs in preterm infants, as their SP-A concentrations are ${ }^{\left.10 w^{11,20}\right)}$. A third cell-damaging pathway induced by Ureaplasma is the hydrolyzation of urea to generate ATP. Hydrolyzation of urea produces ammonia and changes in $\mathrm{pH}$. In combination with the release of serine/threoninekinase, hydrogen peroxide and phospholipases degrade the cell membrane of pneumocytes; these changes are cytotoxic ${ }^{12)}$ (Figure 1).

Because of its cytotoxic behavior, the place of exposure to Ureaplasma is essential. Positive tracheal aspiration and mechanical ventilation at any duration increases the risk of developing BPD by 7.9 fold compared to positive nasopharyngeal samples and mechanical ventilation ${ }^{10)}$. The time point of exposure is also crucial. Postnatal Ureaplasma colonized preterm infants may show less severe respiratory distress symptoms, but show already signs of emphysematous changes of BPD in early radiographic examination ${ }^{12}$. This indicates that early treatment of maternal Ureaplasma infections in utero should be considered or that prophylactic treatment may be important for preventing the development of BPD. Despite these finding regarding the pathophysiologic behavior of Ureaplasma, the exact influence on the development of BPD remains unknown.

\section{UREAPLASMA AND DIAGNOSIS}

In premature infants, analysis of Ureaplasma spp. in the tracheal aspirate can be performed via culture identification assay and/or polymerase chain reaction (PCR) ${ }^{12,21)}$. Culture remains the gold standard for Ureaplasma spp. Because these organisms lack a cell wall and are susceptible to drying and heat, proper sample collection and processing are essential to avoid false-negative results. Tracheal aspirates or nasopharyngeal swabs should be directly inoculated into transport media for transport on ice to the laboratory. New techniques, such as colometric assays or molecular diagnostic methods, are available for detecting Ureaplasma spp.; the colometric approach is less sensitive than PCR is but is much less time consuming. Molecular assays involving gel based conventional PCR, real-time PCR or multiplex PCR cannot distinguish between living and dead organisms and yield no antibiotic susceptibility. The latest identification method is a deoxyribonucleic acid (DNA) chip assay that also cannot assess antibiotic susceptibility ${ }^{12}$. However, no neonatal clinical trials have been performed using this method.

Recently, a study analyzed gastric fluid versus amniotic fluid of preterm infants for the identification of intra uterine infections ${ }^{22)}$. Amniotic fluid analysis showed however better results. Gastric fluid analysis shows $100 \%$ specificity in the identification, but if amniotic fluid tested positive for Ureaplasma and gastric fluid was negative, there was still a higher rate of neonatal mortality. 
There was no significant difference if both amniotic fluid and gastric fluid were tested positive ${ }^{22)}$.

In summary, the best diagnostic tool and approach remains to be identified. With the current development of less invasive surfactant administration that avoids intubation, less tracheal aspirates will be available. Therefore, cultures from other materials, such as nasopharyngeal swabs, ear swabs or gastric fluids, are necessary. However, no data are available supporting the practical use and reliability of these swabs for testing premature infants. This may be a limitation to diagnosis and therefore limit the prevention and treatment of BPD.

\section{UREAPLASMA AND TREATMENT}

In analyzing the characteristics and pathophysiologic behavior of Ureaplasma spp., therapeutic and prophylactic treatment of BPD appear to be important. Treatment of these infections may reduce the incidence, severity, morbidity and mortality of BPD.

However, how and when do we treat Ureaplasma spp. when they are detected? The actual choice of antibiotics is not straight forward. Macrolides are currently the most promising antibiotics available for both prophylactic and therapeutic use. A series of randomized controlled trials, systematic review and metaanalysis evaluated the current evidence on the use of macrolides. Macrolides not only have an anti-bacterial effect, but also inhibit neutrophil migration reducing IL-6, IL-8 and ICAM-1 production by bronchial epithelial cells ${ }^{7}$. In vitro and in vivo experimental models also confirmed that macrolides are preferentially concentrated in the lung epithelial lining fluid and alveolar macrophages where they show antimicrobial activity against Ureaplasma spp. Treatment with these antibiotics may therefore enhance Ureaplasma clearance and reduce the risk of developing $\mathrm{BPD}^{19)}$. However, half of the $U$. urealyticum strains were shown to be resistant against erythromycin ${ }^{10)}$. A Cochrane review in 2003 revealed that treatment of intubated preterm infants at risk for BPD or colonized or infected with Ureaplasma with erythromycin failed to prevent the development of BPD ${ }^{23)}$. In vitro studies showed that clarithromycin, josamycin and azithromycin were the most potent macrolides against Ureaplasma, compared to erythromycin. Higher drug concentration in lung epithelium were observed in these studies ${ }^{10,24-26)}$.

A meta-analysis by Nair et al. demonstrated a significant reduction in BPD and the composite outcome after prophylactic azithromycin therapy ${ }^{7)}$. Pharmacokinetic studies by Hassan et al. ${ }^{27,28)}$ showed that azithromycin at a dose of $20 \mathrm{mg} / \mathrm{kg} /$ day for 3 days was safe and resulted in better clearance of Ureaplasma $s p p$. compared to results of Ballard in $2011^{26)}$. The limitations of these studies was that the dosage was empirically chosen and not based on pharmacokinetic studies. However, there is limited information regarding the pharmacokinetics and potential side effects in neonates. Therefore, further studies of the use of azithromycin in neonates are necessary. A meta-analysis by Nair et al. ${ }^{7)}$ on the prophylactic or therapeutic use of clarithromycin was not possible because there was only one small study performed and published.

Recently, Resch et al. found that there was still a significant association with pulmonary short- and long term morbidity and mild cerebral impairment despite treatment with macrolides antibiotics of preterm infants who were colonized with Urea plasma $^{29)}$. This association of long term adverse outcome requires further analysis.

A new fluoroketolide antimicrobial, solithromycin, has shown promising efficacy in sheep models, where it crosses the placenta and eradicates Ureaplasma spp. infections of the fetuses and amniotic fluid $^{12)}$. Solithromycin is acid stable, and well tolerated, and shows superior tissue accumulation and efficient maternalto-fetal transfer. Thus far, all Ureaplasma spp. tested were sensitive to solithromycin, indicating its promise as an agent for treating intra uterine infections. However, it has not yet been administered in pregnancy and the necessary safety studies in pregnant women have not been conducted ${ }^{25,30,311}$.

Recent studies showed that Ureaplasma spp. self-replicate, and can form a biofilm ${ }^{32}$. Although the biofilm shows low virulence, these organisms evade the host immune response and increase the resistance to antibiotic treatment. The exact mechanism remains unknown but complicates treatment options with anti-

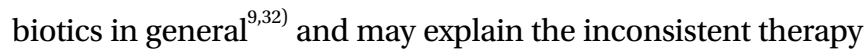
effects.

In summary, there is limited evidence regarding the use of macrolides for the therapeutic treatment of Ureaplasma colonization or the prophylactic use for preventing BPD in preterm infants ${ }^{7}$. The time point, drug, and dose of antibiotic treatment needs to be studied in future clinical trials to optimize the outcome. Ideally, the antibiotic treatment should start in utero. 


\section{SUMMARY}

Ureaplasma plays an important role in the development of BPD. The association between infections and colonization of Ureaplasma spp. with the risk of development of BPD has been supported by several meta-analyses. Therefore, identifying Ureaplasma spp. in preterm infants is important, but challenging. Culturing techniques are the standard procedure, with the highest sensitivity and specificity for culturing in tracheal aspirates. For clinical use, PCR techniques are effective for detecting colonization or infections with Ureaplasma spp. With the increasing use of less invasive ventilation techniques, accurate diagnosis and therefore interpreting the clinical relevance of the presence of Ureaplasma is challenging. However, when colonization or active infection is detected, treatment to prevent the development of BPD is a logical choice. However, the choice among the limited drug options is controversial. The time of starting the treatment, the type of treatment (prophylactic or therapeutic), and the duration for which treatment should be offered are also unclear. Data concerning the pharmacokinetics, dosage, duration of therapy, microbiological efficacy, antimicro bial resistance and safety of macrolides antimicrobials in preterm neonates are limited. Despite the limited options, macrolides are the promising antimicrobial agents currently available for use in neonatal Ureaplasma infections. Studies have shown that either prophylactic or therapeutic treatment with erythromycin will infrequently eradicate Ureaplasma spp. from the lower respiratory tract without significantly affecting the respiratory outcome. Half of the $U$. urealyticum strains were shown to be resistant against erythromycin. Limited data are available regarding clarithromycin, and therefore no definite conclusion can be drawn or advice can be given. Meta-analysis demonstrated a significant reduction in BPD and the composite outcome of BPD after prophylactic azithromycin therapy, but there is limited evidence and knowledge of the pharmacokinetics of azithromycin and its use in treatment. Research is still in progress. Animal studies have shown promising results concerning a new macrolide, solithromycin, but further in vivo research and clinical trials are necessary.

\section{CONFLICTS OF INTEREST AND SOURCE OF FUNDING}

The authors declare no conflict of interest. Matthias Hütten was supported by a grant from Deutsche Forschungsgemeinschaft (DFG, project number Ku 1403/6-1). The authors declare no other financial support.

\section{REFERENCES}

1) Poindexter BB, Feng R, Schmidt B, Aschner JL, Ballard RA, Hamvas A, et al. Comparisons and limitations of current definitions of bronchopulmonary dysplasia for the prematurity and respiratory outcomes program. Ann Am Thorac Soc 2015; 12:1822-30.

2) Northway WH, Jr., Rosan RC, Porter DY. Pulmonary disease following respirator therapy of hyaline-membrane disease. Bronchopulmonary dysplasia. N Engl J Med1967;276:357-68.

3) Hütten MC, Wolfs TG, Kramer BW. Can the preterm lung recover from perinatal stress? Mol Cell Pediatr 2016;3:15.

4) Ehrenkranz RA, Walsh MC, Vohr BR, Jobe AH, Wright LL, Fanaroff AA, et al; National Institutes of Child Health and Human Development Neonatal Research Network. Validation of the National Institute of Health consensus definition of bronchopulmonary dysplasia. Pediatrics 2005;116:1353 -60.

5) Schmidt B, Asztalos EV, Roberts RS, Robertson CM, Sauve RS, Whitfield MF. Impact of bronchopulmonary dysplasia, brain injury, and severe retinopathy on the outcome of extremely lowbirth-weight infants at 18 months: results from the trial of indomethacin prophylaxis in preterms. JAMA 2003;289:1124-9.

6) Kotecha S, Hodge R, Schaber JA, Miralles R, Silverman M, Grant WD. Pulmonary Ureaplasma urealyticum is associated with the development of acute lung inflammation and chronic lung disease in preterm infants. Pediatr Res 2004;55:61-8.

7) Nair V, Loganathan P, Soraisham AS. Azithromycin and other macrolides for prevention of bronchopulmonary dysplasia: a systematic review and meta-analysis. Neonatology 2014;106: 337-47.

8) Moss TJ, Knox CL, Kallapur SG, Nitsos I, Theodoropoulos C, Newnham JP, et al. Experimental amniotic fluid infection in sheep: effects of Ureaplasma parvum serovars 3 and 6 on preterm or term fetal sheep. Am J Obstet Gynecol 2008;198:122.e18.

9) Wang EE, Ohlsson A, Kellner JD. Association of Ureaplasma urealyticum colonization with chronic lung disease of prematurity: results of a meta-analysis. J Pediatr 1995;127:640-4. 
10) Sung TJ, Xiao L, Duffy L, Waites KB, Chesko KL, Viscardi RM. Frequency of ureaplasma serovars in respiratory secretions of preterm infants at risk for bronchopulmonary dysplasia. Pediatr Infect Dis J 2011;30:379-83.

11) Schelonka RL, Katz B, Waites KB, Benjamin DK, Jr. Critical appraisal of the role of Ureaplasma in the development of bronchopulmonary dysplasia with metaanalytic techniques. Pediatr Infect Dis J 2005;24:1033-9.

12) Viscardi RM, Kallapur SG. Role of Ureaplasma respiratory tract colonization in bronchopulmonary dysplasia pathogenesis. Current concepts and update. Clin Perinatol 2015;42:719-38.

13) Lowe J, Watkins WJ, Edwards MO, Spiller OB, Jacqz-Aigrain E, Kotecha SJ, et al. Association between pulmonary ureaplasma colonization and bronchopulmonary dysplasia in preterm infants. Updated systematic review and meta-analysis. Pediatr Infect Dis J 2014;33:697-702.

14) Jonsson B, Rylander M, Faxelius G. Ureaplasma urealyticum, erythromycin and respiratory morbidity in high risk preterm neonates. Acta Pediatr 1998;87:1079-84.

15) Olomu IN, Hecht JL, Onderdonk AO, Allred EN, Leviton A. Perinatal correlates of Ureaplasma urealyticum in placenta parenchyma of singleton pregnancies that end before 28 weeks of gestation. Pediatrics 2009;123:1329-36.

16) Katz B, Patel P, Duffy L, Schelonka RL, Dimmitt RA, Waites KB. Characterization of ureaplasmas isolated from preterm infants with and without bronchopulmonary dysplasia. J Clin Microbiol 2005;43:4852-4.

17) Li YH, Brauner A, Jonsson B, van der Ploeg I, Soder O, Holst M, et al. Ureaplasma urealyticum-induced production of proinflammatory cytokines by macrophages. Pediatr Res 2000;48: 114-9.

18) Li YH, Chen M, Brauner A, Zheng C, Skov Jensen J, Tullus K. Ureaplasma urealyticum induces apoptosis in human lung epithelial cells and macrophages. Biol Neonate 2002;82:166-73.

19) Kallapur SG, Kramer BW, Jobe AH. Ureaplasma and BPD. Semin Perinatol 2013;37:94-101.

20) Okogbule-Wonodi AC, Chesko KL, Famuyide ME, Viscardi RM. Surfactant protein-A enhances ureaplasmacidal activity in vitro. Innate Immun 2011;17:145-51.

21) Cultrera R, Seraceni S, Germani R, Contini C. Molecular evidence of Ureaplasma urealyticum and Ureaplasma parvum colonization in preterm infants during respiratory distress syndrome. BMC Infect Dis 2006;6:166.

22) Kim SM, Romero R, Lee J, Chaemsaithong P, Docheva N, Yoon $\mathrm{BH}$. Gastric fluid versus amniotic fluid analysis for the identification of intra-amniotic infection due to Ureaplasma species. J Matern Fetal Neonatal Med 2016;29:2579-87.
23) Mabanta CG, Pryhuber GS, Weinberg GA, Phelps DL. Erythromycin for the prevention of chronic lung disease in intubated preterm infants at risk for, or colonized or infected with Ureaplasma urealyticum. Cochrane Database Syst Rev 2003: CD003744. doi:10.1002/14651858.CD003744.

24) Krausse R, Schubert S. In-vitro activities of tetracyclines, macrolides, fluoroquinolones and clindamycin against Mycoplasma hominis and Ureaplasma ssp. isolated in Germany over 20 years. Clin Microbiol Infect 2010;16:1649-55.

25) Ozdemir R, Erdeve O, Dizdar EA, Oguz SS, Uras N, Saygan S, et al. Clarithromycin in preventing bronchopulmonary dysplasia in Ureaplasma urealyticum-positive preterm infants. Pediatrics 2011;128:e1496-501.

26) Ballard HO, Shook LA, Bernard P, Anstead MI, Kuhn R, Whitehead V, et al. Use of azithromycin for the prevention of bronchopulmonary dysplasia in preterm infants: a randomized, double-blind, placebo controlled trial. Pediatr Pulmonol 2011; 46:111-8.

27) Hassan HE, Othman AA, Eddington ND, Duffy L, Xiao L, Waites KB, et al. Pharmacokinetics, safety, and biologic effects of azithromycin in extremely preterm infants at risk for ureaplasma colonization and bronchopulmonary dysplasia. J Clin Pharmacol 2011;51:1264-75.

28) Merchan LM, Hassan HE, Terrin ML, Waites KB, Kaufman DA, Ambalavanan N, et al. Pharmacokinetics, microbial response, and pulmonary outcomes of multidose intravenous azithromycin in preterm infants at risk for Ureaplasma respiratory colonization. Antimicrob Agents Chemother 2015;59:570-8.

29) Resch B, Gutmann C, Reiterer F, Luxner J, Urlesberger B. Neonatal Ureaplasma urealyticum colonization increases pulmonary and cerebral morbidity despite treatment with macrolide antibiotics. Infection 2016;44:323-7.

30) Miura Y, Payne MS, Keelan JA, Noe A, Carter S, Watts R, et al. Maternal intravenous treatment with either azithromycin or solithromycin clears Ureaplasma parvum from the amniotic fluid in an ovine model of intrauterine infection. Antimicrob Agents Chemother 2014;58:5413-20.

31) Furfaro LL, Spiller OB, Keelan JA, Payne MS. In vitro activity of solithromycin and its metabolites, CEM-214 and N-acetylCEM-101, against 100 clinical Ureaplasma spp. isolates compared with azithromycin. Int J Antimicrob Agents 2015;46: 31924.

32) Garcia-Castillo M, Morosini MI, Galvez M, Baquero F, del Campo R, Meseguer MA. Differences in biofilm development and antibiotic susceptibility among clinical Ureaplasma urealyticum and Ureaplasma parvum isolates. J Antimicrob Chemother 2008;62:1027-30. 